\title{
Excitonic condensation of strongly correlated electrons: the case of $\mathrm{Pr}_{0.5} \mathrm{Ca}_{0.5} \mathrm{CoO}_{3}$
}

\author{
Jan Kunes* and Pavel Augustinský \\ Institute of Physics, Academy of Sciences of the Czech Republic, \\ Cukrovarnická 10, 162 53 Praha 6, Czech Republic
}

\begin{abstract}
We use a combination of dynamical mean-field model calculations and LDA+U material specific calculations to investigate the low temperature phase transition in the compounds from the $\left(\mathrm{Pr}_{1-y} \mathrm{R}_{y}\right)_{x} \mathrm{Ca}_{1-x} \mathrm{CoO}_{3}(\mathrm{R}=\mathrm{Nd}, \mathrm{Sm}, \mathrm{Eu}, \mathrm{Gd}, \mathrm{Tb}, \mathrm{Y})$ family (PCCO). The transition, marked by a sharp peak in the specific heat, leads to an exponential increase of dc resistivity and a drop of the magnetic susceptibility, but no order parameter has been identified yet. We show that condensation of spin-triplet, atomic-size excitons provides a consistent explanation of the observed physics. In particular, it explains the exchange splitting on the Pr sites and the simultaneous Pr valence transition. The excitonic condensation in PCCO is an example of a general behavior expected in certain systems in the proximity of a spin-state transition.
\end{abstract}

PACS numbers:

The $\mathrm{R}_{x} \mathrm{~A}_{1-x} \mathrm{CoO}_{3} \quad(\mathrm{R}=\mathrm{La}, \ldots$, and $\mathrm{A}=\mathrm{Ca}, \mathrm{Sr}$, $\mathrm{Ba}$ ) series exhibits a variety of phenomena including thermally and doping driven spin-state crossover, metal-insulator crossover, magnetic ordering or nanoscopic inhomogeneities. The root cause of the rich physics are quasi-degenerate Co $3 d$ atomic multiplets and their interaction with the crystal lattice or doped charge carriers. The $\left(\mathrm{Pr}_{1-y} \mathrm{R}_{y}\right)_{x} \mathrm{Ca}_{1-x} \mathrm{CoO}_{3}(\mathrm{R}=\mathrm{Nd}, \mathrm{Sm}, \mathrm{Eu}, \mathrm{Gd}, \mathrm{Tb}$, $\mathrm{Y})$ family is unique among the cobaltites. A decade ago, Tsubouchi et al [1, 2] observed a metal-insulator transition in $\mathrm{Pr}_{0.5} \mathrm{Ca}_{0.5} \mathrm{CoO}_{3}$ associated with a drop of magnetic susceptibility and a sharp peak in the specific heat indicating the collective nature of the transition. Subsequently, the transition was observed in other PCCO materials with $x$ and $y$ in the ranges $0.2-0.5$ and $0-0.3$, respectively $3-5]$. Despite the evidence for a continuous, or very weakly first order, phase transition and the experimental effort [6] no long-range order could be identified. The PCCO materials in this respect resemble the much famous hidden order prototype $\mathrm{URu}_{2} \mathrm{Si}_{2}[7]$. An important step towards understanding of the transition in $\mathrm{PCCO}$ was made by observation of $\mathrm{Pr}^{3+} \rightarrow \mathrm{Pr}^{4+}$ valence transition which take place simultaneously. $[6,8]$. Another clue to the nature of the PCCO hidden order is the exchange splitting of the $\operatorname{Pr}^{4+}$ Kramers ground state in the absence of ordered magnetic moments [4, 6, 9].

The basic features to be captured by a theory of the PCCO hidden order are: i) substantial increase of resistivity below $T_{c}, i i$ ) the sharp peak in the specific heat at $T_{c}$, iii) the drop of the magnetic susceptibility and the departure from the Curie-Weiss behavior of the Co moments below $\left.T_{c}, i v\right)$ the $\operatorname{Pr}$ valence transition, $v$ ) the exchange splitting of the
$\operatorname{Pr}^{4+}$ Kramers doublet in the absence of ordered magnetic moments. More subtle effects include the increase of $T_{c}$ with pressure [3], the lattice response consisting primarily in reduction of the Co-O-Co angle below $T_{c}$ [3], and the apparent softness of the exchange field on $\mathrm{Pr}$ and the lack of a clear x-ray signature of the spin-state transition [6, 10].

In this Letter we explain the physics of $\mathrm{PCCO}$ by formation of excitonic condensate (EC). Motivated by observation of excitonic instability of correlated electrons close to a spin-state transition [1], we have performed two types of investigations. First, we have studied the EC phase in a minimal model using the dynamical mean-field theory (DMFT) [12] and calculated temperature $(T)$ dependencies of the various physical quantities across the transition. Second, we have obtained a $T=0 \mathrm{EC}$ solution for PCCO using the density-functional LDA+U method [13, 14].

Two-orbital Hubbard model (11) captures the competition between the atomic high-spin (HS) and lowspin (LS) states and thus provides a minimal description of a solid with a spin-state transition.

$$
\begin{aligned}
& H=\frac{\Delta}{2} \sum_{i, \sigma}\left(n_{i \sigma}^{a}-n_{i \sigma}^{b}\right)+\sum_{\langle i j\rangle, \sigma}\left(t_{a} a_{i \sigma}^{\dagger} a_{j \sigma}+t_{b} b_{i \sigma}^{\dagger} b_{i \sigma}\right) \\
& +U \sum_{i, \alpha=a, b} n_{i \uparrow}^{\alpha} n_{i \downarrow}^{\alpha}+U^{\prime} \sum_{i, \sigma} n_{i \sigma}^{a} n_{i \bar{\sigma}}^{b}+\left(U^{\prime}-J\right) \sum_{i, \sigma} n_{i \sigma}^{a} n_{i \sigma}^{b} .
\end{aligned}
$$

Here $a_{i \sigma}^{\dagger}, b_{i \sigma}^{\dagger}$ are the creation operators of fermions with spin $\sigma=\uparrow, \downarrow$ in orbitals $a$ and $b$ on the site $i$ of a square lattice, and $n_{i \sigma}^{a, b}$ are the corresponding occupation number operators. The DMFT calculations using the impurity solver of Werner et al. [15] were performed for $U^{\prime}=U-2 J, U=4$, $J=1, t_{a}=0.4118, t_{b}=-0.1882$ and $\Delta=3.40$, 

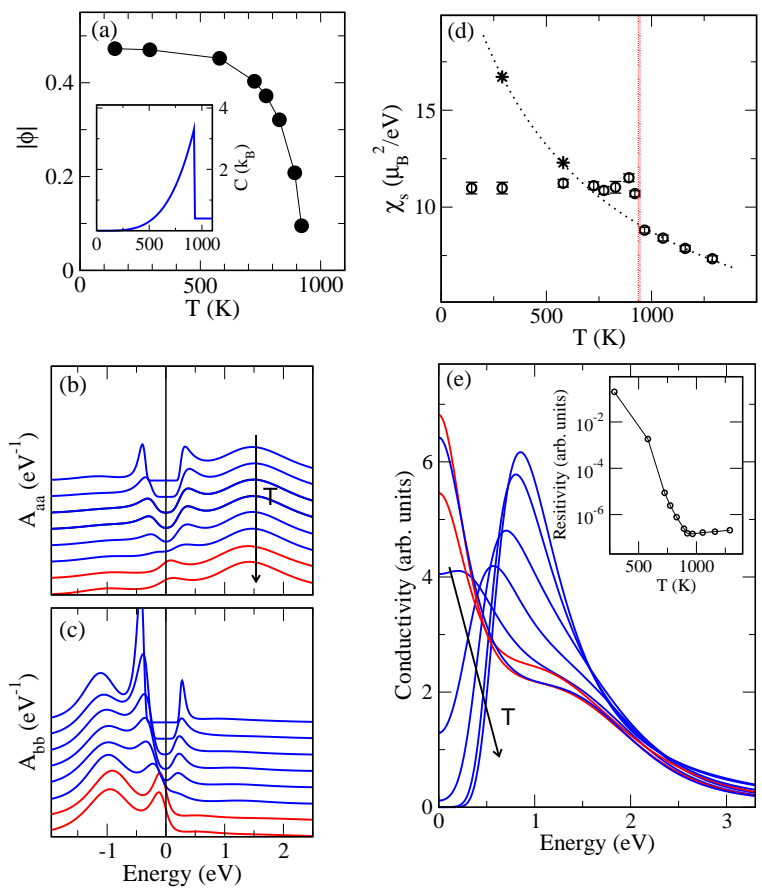

FIG. 1: The DMFT results for fixed particle density $n=2$. (a) The magnitude of the order parameter $|\phi(T)|$. The inset shows specific heat $C(T)$. (b, c) The spectral functions $A_{a a}(\omega)$ and $A_{b b}(\omega)$ at $T=1160,968,921,892$, $829,725,580,290 \mathrm{~K}$. (blue curves for $T<T_{c}$, the red ones for $T>T_{c}$ ). The arrow marks direction of increasing temperature. (e) The corresponding optical conductivity. The inset shows the dc resistivity. (d) The spin susceptibility $\chi_{S}(T)$ (circles with error bars) and $\chi_{S}(T)$ of the constrained normal phase solutions (dotted line).

assuming $\mathrm{eV}$ to be the unit of energy. Details can be found in the Supplemental Material (SM). The model captures the basic features of perovskite cobaltites: nearly degenerate LS and HS atomic states, the energy scales of the bandwidths and the interaction strength, a band gap/overlap being much smaller than the bandwidths, and the dominant CoCo nearest-neighbor hopping on a bipartite lattice preserving the orbital flavor. The main approximation consists in neglecting the actual orbital degeneracy of the $d$-shell.

Linear response calculations [1] predicted the model to exhibit excitonic instability in the magnetic channel. The spin-triplet EC order parameter for the model with $\mathrm{SU}(2)$ symmetric interaction is a vector $\phi_{i}=\sum_{\alpha \alpha^{\prime}=\uparrow, \downarrow} \boldsymbol{\sigma}_{\alpha \alpha^{\prime}}\left\langle a_{i \alpha}^{\dagger} b_{i \alpha^{\prime}}\right\rangle[16$, 17], where $\sigma$ are the Pauli matrices. For the density-density interaction of Hamiltonian (10) $\phi$ is constrained to the $x y$-plane. First, we investigate the model at fixed particle density $n$ of 2 electrons per atom. In
Fig. 17 we show the evolution of the order parameter $\phi$, which was chosen to point in the $x$-direction. The inset shows the specific heat per atom with a typical mean-field shape. Non-zero $\phi_{x}$ is connected to appearance of a spin off-diagonal (anomalous) element of the self-energy (see SM for an example), which opens a gap in the one-particle spectra, Fig. 1b,c. The gap opening is reflected in the behavior of the optical conductivity, Fig. The The Drude peak is suppressed below $T_{c}$ and the dc resistivity, shown in the inset, grows exponentially upon cooling. While there are no ordered moments below $T_{c}$ the spin susceptibility $\chi_{S}(T)$, Fig. 1 $\mathrm{d}$, is strongly affected by the EC transition. In the high- $T$ normal phase, thermally excited HS states lead to Curie-Weiss $\chi_{S}(T)$. While HS states are present in the EC phase, they are not free. The anomalous self-energy gives rise to an on-site hybridization between the LS and HS states which results in a $T$-independent Van Vleck $\chi_{S}(T)$. The sign of the change of $\chi_{S}(T)$ at $T_{c}$ depends on details of the system, in particular, a reduction of $T_{c}$ by doping, as discussed below, leads to the same sign of $\chi_{S}(T)$ change as in the experiment. The fact that HS population does not vanish in the EC phase can explain the lack of changes in the x-ray spectra [6] typical for the spin-state transition.

The Co bands of PCCO differ from the above model in an important aspect. They are hole doped in the normal state and their filling changes due to the $\mathrm{Pr}^{3+} \rightarrow \operatorname{Pr}^{4+}$ valence transition. The isostructural valence transition points to a near degeneracy of the $f^{2}$ and $f^{1}$ states of the $\operatorname{Pr} 4 f$ shell. The Pr ions therefore act as a charge reservoir providing electrons to the Co bands and can be modeled by fixing the chemical potential $(\mu)$ in the above calculations. In the following we present model results obtained with fixed $\mu$. The particle density $n(T)$ in the normal phase is very weakly $T$-dependent and thus can be used to label the different choices of $\mu$. In Fig. 2 we show $|\phi|$ for dopings between 0.03 and 0.12 holes per atom. Doping away from the half filling leads to a reduction of $T_{c}$. Unlike in the normal phase, $n(T)$ varies considerably below $T_{c}$. With decreasing $T$ the system draws electrons from the reservoir, a process controlled by competition between the condensation energy, favoring an equal number of $a$-electrons and $b$-holes, and the energy of adding electrons from the reservoir. The present theory thus provides a simple connection between the Pr valence change and the EC transition, and explains why these happen simultaneously [6]. The evolution of the one-particle spectra at fixed $\mu$ (see SM) is similar to Fig. 1b,c although the spectrum becomes fully gapped only at half filling. The be- 


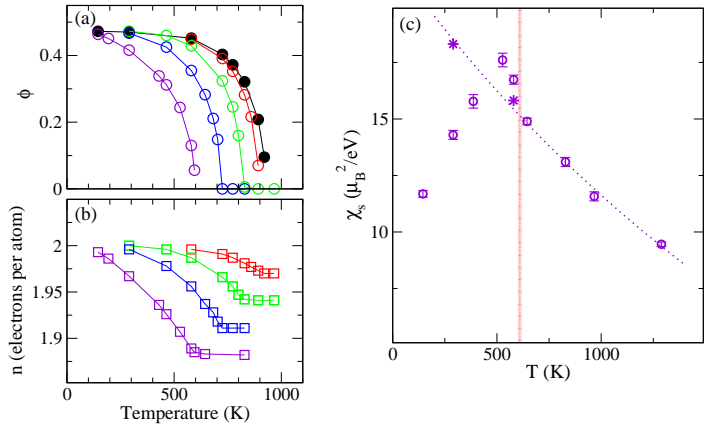

FIG. 2: (a) The magnitude of the order parameter $|\phi(T)|$ for various fixed chemical potentials (The $|\phi(T)|$ for $n=2$ taken from Fig. 1 is marked by black circles). (b) The corresponding particle densities $n$ as functions of temperature. The curves correspond to the doping of 0.03 (red), 0.06 (green), 0.09 (blue) and 0.12 (violet) holes per atom in the normal phase. (c) The susceptibility $\chi_{S}(T)$ for the 0.12 hole doping. The symbols have the same meaning as in Fig. 1.

havior of the $\chi_{S}(T)$ for fixed $\mu$ is shown in Fig. 2r.

The model calculations capture the features $i-i v$. The $i$-iii are generic features of the EC transition in a half-filled system that survive to a doped material kept at fixed $\mu$. The feature $i v$ is accounted for by treating the Pr ions as a charge reservoir for the Co bands. There are several limitations associated with the DMFT method as well as the model itself. The mean-field character of the method is responsible for the extremely asymmetric peak in the specific heat $C(T)$ as well as the kink in $n(T)$ at $T_{c}$. The experimental $C(T)$ and $n(T)[\underline{6}$ ] do not exhibit this pronounced asymmetry which can be explained by short-range EC correlations above $T_{c}$. The model also ignores the change of the lattice below $T_{c}$ consisting in bending of $\mathrm{Co}-\mathrm{O}-\mathrm{Co}$ without changing the Co-O bondlength. It enhances the $e_{g}$ - $t_{2 g}$ hopping, which provides a positive feedback to the EC transition. The transition with lattice taken into account is therefore expected to be sharper, perhaps even weakly first order, than in a purely electronic model.

In order to test the EC scenario in a more realistic setting and to address the feature $v$ we have performed a material specific calculation using $\mathrm{LDA}+\mathrm{U}$ method. It roughly amounts to a $T=0$ static meanfield solution for Hamiltonian including all electronic orbitals, the experimental crystal structure and unrestricted hopping. Such calculation can answer the question whether the EC order in PCCO is plausible. The ability of the method to capture complex long-range orders was demonstrated by Cricchio et al. [18, 19] on $\mathrm{URu}_{2} \mathrm{Si}_{2}$ and $\mathrm{LaFeAsO}$.
Before presenting the results for the orthorhom 3 bic PCCO structure we discuss symmetry aspects of EC in a cubic crystal. As in the model, the Hund's coupling selects the spin part of the order parameter to be a triplet. The orbital part describes a pair of an $e_{g}$-electron and a $t_{2 g}$-hole, which transforms as $E_{g} \times T_{2 g}=T_{2 g}+T_{1 g}$ representation under the cubic symmetry operations. General considerations suggest that only $T_{1 g}$ pairs, $d_{x y} \otimes d_{x^{2}-y^{2}}, d_{x z} \otimes d_{x^{2}-z^{2}}$ and $d_{y z} \otimes d_{y^{2}-z^{2}}$ can condense. The electrons and holes forming a $T_{1 g}$ pair have large hopping amplitudes along the same 'in-plane' directions, while the electrons and holes forming a $T_{2 g}$ pair of the form $d_{x y} \otimes d_{z^{2}}$ maximize their hoppings in perpendicular directions, which is detrimental to the condensation. Moreover, the electron-hole bonding is stronger for a $T_{1 g}$ than for a $T_{2 g}$ pair. The EC order in a cubic symmetry is thus characterized by nine parameters $\phi_{\beta}^{\alpha}$, where $\alpha$ runs over the three Cartesian spin components and $\beta$ over three $T_{1 g}$ orbital components. The anomalous part of the Co $3 d$ occupation matrices in terms of $\phi_{\beta}^{\alpha}$ can be found in SM. We have verified that the numerical $\mathrm{LDA}+\mathrm{U}$ solutions have these symmetry properties by performing a series of calculations in cubic perovskite structure, which will be reported separately.

The LDA $+\mathrm{U}$ calculations for PCCO were performed in the structure of Ref. 8 with a unit cell containing four Co sites. On-site interaction parametrized with $\mathrm{U}=4 \mathrm{eV}$ and $\mathrm{J}=1 \mathrm{eV}$ was assumed for the Co $3 d$ shells. All Pr ions were assumed to be in the $4+$ state, which was enforced by constraining the $f^{1}$ occupancy in so called core treatment of the $\operatorname{Pr} 4 f$ states. We found a stable EC solution with the total energy $230 \mathrm{meV}$ per formula unit lower than the normal state one. The EC was detected by appearance of spin-triplet terms in the Co-3d occupation matrix. Reflecting the approximate cubic symmetry of the Co sites, the orbital part of the anomalous terms is dominated by the $T_{1 g}$ components. The order parameter of the present solution can be written as a product $\phi_{\beta}^{\alpha}=\varphi_{\beta} \otimes e_{S}^{\alpha}$ of a spin vector $e_{S}^{\alpha}$ pointing in arbitrary direction, but common to all Co sites, and an orbital pseudo-vector $\varphi_{\beta}$, shown in Table प. The product form of $\phi_{\beta}^{\alpha}$ with real elements results in the collinear spin-density distribution shown in Fig. 3. Inspection of $\varphi_{\beta}$ for symmetry related Co sites reveals an odd parity of the order parameter under the mirror image $\sigma_{h}$ by a plane perpendicular to the $c$-axis. The EC solution does not exhibit ordered local moments $\left(|\mathbf{m}|<0.03 \mu_{B}\right.$ inside Wien2k atomic spheres). The orbital resolved spectral functions can be found in SM.

Next, we address $v$ ) the exchange splitting of the 
TABLE I: The orbital parts of the EC order parameter for the four Co atoms the unit cell of PCCO in local coordinates tied to the $\mathrm{CoO}_{6}$ octahedra. The sites 1-2 and 3-4 are connected by $\sigma_{h}$ symmetry.

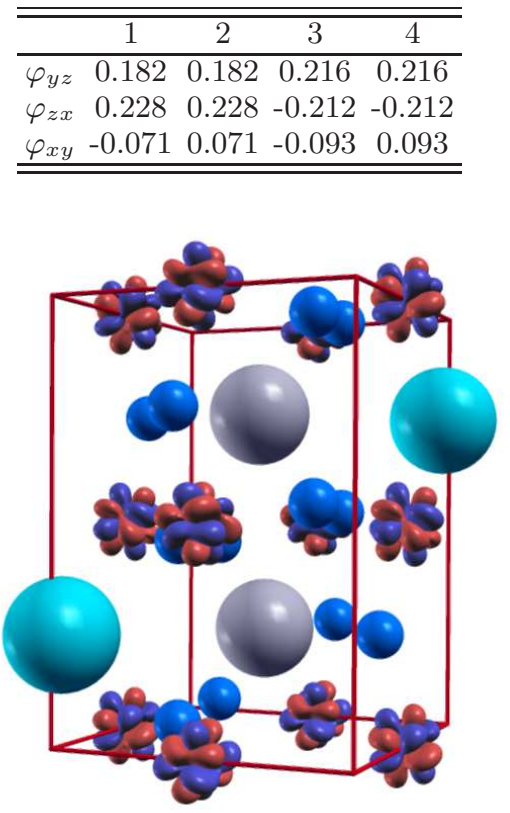

FIG. 3: The distribution of the collinear spin density (red and blue correspond to positive and negative sign) around Co atoms in PCCO with O (blue), Ca (light blue) and $\operatorname{Pr}$ (grey).

Kramers ground state of the $\mathrm{Pr}^{4+}$ ion. The EC with real $\phi_{\beta}^{\alpha}$ breaks the time reversal symmetry. However, we have to show that this symmetry breaking is felt by the Pr moments. Microscopic analysis based on a multi-band Kondo impurity model can be found in SM. Here we use direct numerical calculation. To estimate the exchange splitting arising from the $4 f$ ligand hybridization we diagonalize the Kohn-Sham Hamiltonian of the EC solution with $\operatorname{Pr} 4 f$ orbitals included (with $E_{4 f}$ inside the gap). This approach mimics the effect of the $f^{1} \rightarrow f^{2} \underline{L}$ virtual excitation [20]. The calculated $4 f$ spectrum is shown in Fig. 4. The exchange splitting induced by the EC order is clearly visible on top of the dominant spinorbit and crystal-field splitting. The $4 f$ spin-orbit coupling (SOC) is crucial. As shown in the inset of Fig. 4, the EC order that is odd under the mirror image $\sigma_{h}$ does not couple to individual $4 f$ crystal-field states (without $4 f \mathrm{SOC}$ ), which are either $\sigma_{h}$-odd or $\sigma_{h^{-}}$-even. It is only the SOC, which mixes the $\sigma_{h^{-}}$ odd and $\sigma_{h}$-even $4 f$ functions and thus allows the exchange splitting (see SM for more detail). The exchange splitting of the order of $10 \mathrm{meV}$ overestimates the experimental values of a few Kelvin. This

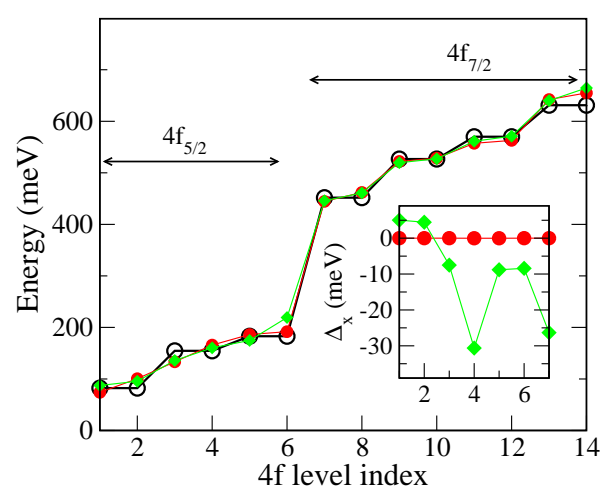

FIG. 4: The spectrum of the $\operatorname{Pr} 4 f$ states: no EC order (black), the self-consistent LDA $+\mathrm{U}$ solution with the $\sigma_{h}$-odd order parameter (red), with an artificial order parameter of the same magnitude containing a $\sigma_{h}$-even contribution (green). The inset shows the exchange splitting of the $4 f$ levels for the same order parameters when spin-orbit coupling is not included.

is not surprising given the approximations involved, in particular the mean-field treatment of the Pr $4 f$ shell which in reality presents a complicated quantum impurity problem.

Spin-triplet excitonic condensation provides a comprehensive description of the phase transition observed in the PCCO series. In particular, we are not aware of an alternative theory of the exchange splitting of the $\operatorname{Pr} 4 f$ states. It is not clear at the moment why the excitonic condensation takes place in PCCO, but not in other cobaltites close to stoichiometric filling, e.g. $\mathrm{LaCoO}_{3}$. The answer is related to the nature of the lowest excited states of the Co ion. The $S=2$ states tend to form a solid lattice on the LS background 21, 22], while $S=1$ states are susceptible to the excitonic condensation. A phase separation is another competing alternative in the doped systems.

The low-temperature phase of PCCO is an example of complex multipole order which is detected only through its indirect effects. Unlike $\mathrm{URu}_{2} \mathrm{Si}_{2}$ or LaOFeAs where the hidden order and nematicity arise from Fermi surface nesting [23, 24], PCCO are strongly correlated oxides and the transition here is closer to condensation of preexisting composite bosons. The present mechanism of the excitonic condensation is quite general and therefore it should be possible to find it in other materials exhibiting singlet-triplet spin-state transitions [25].

We acknowledge numerous discussions with $\mathrm{Z}$. Jirák, P. Novák, A. Kauch and D. Vollhardt. The work was supported through the research unit FOR 1346 of the Deutsche Forschungsgemeinschaft and 
the grant 13-25251S of the Grant Agency of the Czech Republic.

* Electronic address: kunes@fzu.cz

[1] S. Tsubouchi, T. Kyômen, M. Itoh, P. Ganguly, M. Oguni, Y. Shimojo, Y. Morii, and Y. Ishii, Phys. Rev. B 66, 052418 (2002).

[2] S. Tsubouchi, T. Kyômen, M. Itoh, and M. Oguni, Phys. Rev. B 69, 144406 (2004).

[3] T. Fujita, T. Miyashita, Y. Yasui, Y. Kobayashi, M. Sato, E. Nishibori, M. Sakata, Y. Shimojo, N. Igawa, Y. Ishii, et al., Journal of the Physical Society of Japan 73, 1987 (2004).

[4] J. Hejtmánek, E. Šantavá, K. Knížek, M. Maryško, Z. Jirák, T. Naito, H. Sasaki, and H. Fujishiro, Phys. Rev. B 82, 165107 (2010).

[5] V. Hardy, F. Guillou, and Y. Brard, Journal of Physics: Condensed Matter 25, 246003 (2013).

[6] J. Hejtmánek, Z. Jirák, O. Kaman, K. Knížek, E. Šantavá, K. Nitta, T. Naito, and H. Fujishiro, The European Physical Journal B 86, 305 (2013).

[7] J. A. Mydosh and P. M. Oppeneer, Rev. Mod. Phys. 83, 1301 (2011).

[8] K. Knížek, J. Hejtmánek, P. Novák, and Z. Jirák, Phys. Rev. B 81, 155113 (2010).

[9] K. Knížek, J. Hejtmánek, M. Maryško, P. Novák, E. Šantavá, Z. Jirák, T. Naito, H. Fujishiro, and C. de la Cruz, Phys. Rev. B 88, 224412 (2013).

[10] J. Herrero-Martín, J. L. García-Muñoz, K. Kvashnina, E. Gallo, G. Subías, J. A. Alonso, and A. J. Barón-González, Phys. Rev. B 86, 125106 (2012).
[11] J. Kuneš and P. Augustinský, Phys. Rev. B 89, 115134 (2014).

[12] A. Georges, G. Kotliar, W. Krauth, and M. J. Rozenberg, Rev. Mod. Phys. 68, 13 (1996).

[13] A. B. Shick, A. I. Liechtenstein, and W. E. Pickett, Phys. Rev. B 60, 10763 (1999).

[14] P. Blaha, K. Schwarz, G. K. H. Madsen, D. Kvasnicka, and J. Luitz, WIEN2K, An Augmented Plane Wave + Local Orbitals Program for Calculating Crystal Properties (Karlheinz Schwarz, Techn. Universität Wien, Austria, 2001).

[15] P. Werner, A. Comanac, L. de' Medici, M. Troyer, and A. J. Millis, Phys. Rev. Lett. 97, 076405 (2006).

[16] T. Kaneko, K. Seki, and Y. Ohta, Phys. Rev. B 85, 165135 (2012).

[17] B. I. Halperin and T. M. Rice (Academic Press, New York, 1968), vol. 21, p. 115.

[18] F. Cricchio, F. Bultmark, O. Grånäs, and L. Nordström, Phys. Rev. Lett. 103, 107202 (2009).

[19] F. Cricchio, O. Grånäs, and L. Nordström, Phys. Rev. B 81, 140403 (2010).

[20] P. Novák, K. Knížek, and J. Kuneš, Phys. Rev. B 87, 205139 (2013).

[21] K. Knížek, Z. Jirák, J. Hejtmánek, P. Novák, and W. Ku, Phys. Rev. B 79, 014430 (2009).

[22] J. Kuneš and V. Křápek, Phys. Rev. Lett. 106, 256401 (2011).

[23] H. Ikeda, M. Suzuki, R. Arita, T. Takimoto, T. Shibauchi, and Y. Matsuda, Nat. Phys. 8, 528 (2012).

[24] R. M. Fernandes, A. V. Chubukov, and J. Schmalian, Nat. Phys. 10, 97 (2014).

[25] G. Khaliullin, Phys. Rev. Lett. 111, 197201 (2013). 